\title{
CONTROL STRATEGIES FOR THE GRID SIDE CONVERTER IN A WIND GENERATION SYSTEM BASED ON A FUZZY APPROACH
}

\author{
NAZIHA HARRABI ${ }^{a, *}$, MAHER KHARRAT $^{a}, \operatorname{ABDEl~AITOUCHE}^{b}$, MANSOUR SOUISSI $^{a}$ \\ ${ }^{a}$ Laboratory of Sciences and Techniques of Automatic Control and Computer Engineering \\ National Engineering School of Sfax, 3072, Road Soukra km 4, Sfax, Tunisia \\ e-mail: nozha.harrabi@gmail.com \\ ${ }^{b}$ Research Center in Informatics, Signal and Automatic Control in Lille (CRIStAL) \\ University of Lille 1, 59655 Villeneuve d'Ascq Cedex, Lille, France
}

\begin{abstract}
Two techniques for the control of a grid side converter in a wind energy conversion system. The system is composed of a fixed pitch angle wind turbine followed by a permanent magnet synchronous generator and power electronic converters AC-DC-AC. The main interest is in how to control the inverter in order to ensure the stability of the DC link voltage. Two control methods based on the fuzzy approach are applied and compared. First, a direct Mamdani fuzzy logic controller is presented. Then, a T-S fuzzy controller is elaborated based on a T-S fuzzy model. The Lyapunov theorem and H-infinity performance are exploited for stability analysis. Besides, the feedback controller gains are determined using linear matrix inequality tools. Simulation results are derived in order to prove the robustness of the proposed control algorithms and to compare their performances.
\end{abstract}

Keywords: wind energy conversion system, DC link, DC-AC converter, Mamdani fuzzy controller, T-S fuzzy controller.

\section{Introduction}

Reducing the fossil-energy environmental impact over the last few decades has encouraged the interest in generating electricity from renewable energy technologies like solar, wind and bio-mass. Especially, wind power plants have been widely spread as a leading alternative power source in industry (Tsoutsos and Stamboulis, 2005). Many factors are combined to increase the interest in wind energy. Among all renewable energy systems, wind energy based systems are the most environmental safe, clean and cost competitive (Babu and Arulmozhivarman, 2013). Although its history goes back more than two centuries ago, this kind of alternative energy began to get more interest only at the beginning of this century (Goudarzi and Zhu, 2013). In spite of renewable and clean aspects of wind power systems, various challenges have to be addressed. In fact, wind energy availability is statistical in nature and highly depending on wind speed fluctuations. Indeed, wind turbines are considered as complex machines working

* Corresponding author under unpredictable conditions and coupled to varying electrical grid with large changing voltages and power flow (Camacho et al., 2011). Consequently, in order to provide reliable operation and high performances, advanced control strategies must be adopted to overcome problems related to wind speed prediction, power control, voltage and frequency regulation, and the like.

A few topologies are used for wind energy conversion systems (see, e.g., Blaabjerg et al., 2012; Mansour et al., 2011). Generally, wind turbine systems can employ various kinds of generators which can be asynchronous or synchronous (Chinchilla et al., 2006; Tapia et al., 2003; Spooner and Williamson, 1996; Kadam and Kushare, 2012). The most common generator is the doubly fed induction generator (DFIG) which is widely reported in literature. In addition, the permanent magnet synchronous generator (PMSG) is considered as a promising technology to apply since it provides various advantages. Unlike asynchronous machines, it does not require the use of a gearbox which reduces a regular maintenance need. Its use seems to attract more attention in wind applications due to its high power 
density, effectiveness and the possibility of getting high energy material at an acceptable price.

The wind turbine-PMSG is generally coupled to the power grid using power electronic converters which allow the power control and voltage adaptation. Two popular configurations are adopted today (Nguyen and Naidu, 2011). The first one comprises a generator side converter (AC-DC) coupled to a DC link and, a grid side converter (DC-AC). The second topology is composed of a rectifier connected to a DC chopper, a DC link and finally, a DC-AC inverter. Power converters must be cooperatively controlled so that DC link voltage can be maintained constant (Zhang and Cheng, 2010). The wind energy conversion system (WECS) considered in this paper is based on the first topology. Since it is connected directly to the grid, grid side converter control, which is the main focus of this paper, is crucial to fulfill the grid requirements. Therefore, many references on grid side converter control in wind applications are revised. For instance, in the works of Skretas and Papadopoulos (2008) as well as Chen et al. (2006), various control modes have been compared and analyzed. The most widely reported in literature is the PI controller. For other instances, several PI based control schemes are presented by Chung et al. (2010) and Kim et al. (2015). Nevertheless, the adjustment of PI controller parameters is cumbersome, particularly in wind power systems which are hard to be expressed by a transfer function. Hence, advanced control algorithms are needed to control power converters so that it is possible to cope with these issues and ensure better performances. These problems can be solved by incorporating the fuzzy logic approach which is known to be suitable for imprecise situations and complex nonlinear systems.

There are few studies dealing with the DC link voltage regulation based on fuzzy logic as in the works of Chen et al. (2000), Farh and Eltamaly (2013) or Dixon et al. (1997). However, it has been realized that the design of fuzzy controllers in control structures dedicated to wind conversion systems to obtain best performances is still a poorly reported topic. Therefore, in this study, this need is addressed by presenting two different design procedures for the DC bus voltage regulation in a grid-connected inverter applying the fuzzy approach. The main interest of this work is in the study of the grid side inverter control for the wind turbine. The objective is to evaluate the proposed T-S fuzzy based model scheme. For this reason, the latter approach will be compared with standard fuzzy logic control in terms of reliability and stability performances. A simple fuzzy logic controller (FLC) based on Mamdani's theory is developed in order to compute the control signals for the inverter. The contribution of the paper is clear in the other control strategy, which is based on Takagi-Sugeno (T-S) fuzzy modeling. The T-S fuzzy control model allows us to describe a nonlinear system by means of linear submodels blended together by membership functions (Nguang and Shi, 2006). Hence, a control design based on parallel distributed compensation (PDC) theory is presented (Wang et al., 1996). Linear matrix inequalities (LMIs) techniques are exploited in order to determine the feedback gains of the designed controller (Boyd et al., 1994).

This study is structured as follows: in the second section, a description of the physical model for the system considered is detailed. Then, in the third section, the operation principle of the control strategy is presented. The design of the direct Mamdani FLC is developed in the fourth section, while Section 5 is reserved for the presentation of the T-S fuzzy control technique. The proposed control method performances are evaluated and compared based on simulation results shown in Section 6. Finally, the paper ends with a set of main conclusions that could be drawn from this work.

\section{System modeling}

The general scheme of the wind generation system is illustrated in Fig. 11 The zone of interest in this study is marked with a dotted line. The main objective of this work is the control of the grid side which comprises a DC link and an IGBT inverter coupled to the grid through an R-L filter. The control of the machine side converter which includes the wind turbine, the generator and the rectifier is outlined by Harrabi et al. $(2015 ; 2016)$.

Converters are controlled by means of complementary switching functions $u_{1}, u_{2}$ and $u_{3}$ which establish the association between the AC and DC sides.

Since this study deals with the average model of the system, we note that $\beta_{r i}$ and $\beta_{o i}$ represent the average values of the switching functions used to control the rectifier and the inverter, respectively, over a period of time $i=1,2,3$. Using the Park transformation, we define $\beta_{r d}$ and $\beta_{r q}$ as the d-q components of $\beta_{r i}$, and $\beta_{o d}$ and $\beta_{o q}$ as the $\mathrm{d}-\mathrm{q}$ components of $\beta_{o i}$ in the $\mathrm{d}-\mathrm{q}$ reference frame.

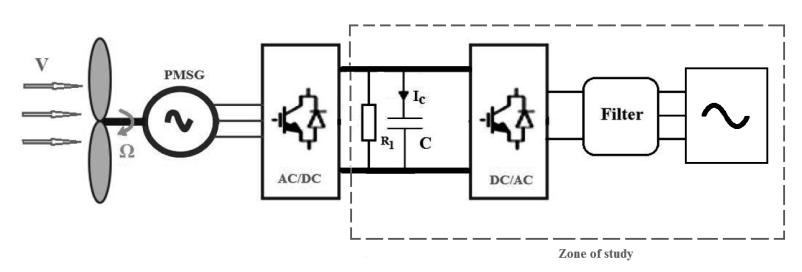

Fig. 1. WECS block diagram.

2.1. DC-link modeling. The back-to-back converter is represented by two voltage source converters connected together through a DC-link as shown in Fig. 11 $C$ re- 
presents the capacitance of the DC-link capacitor and $R_{1}$ is a leakage resistance. $I_{r}$ and $I_{O}$ are respectively the resulting current from the rectifier side and the inverter grid side current. The DC-link voltage $U_{c}$ is regulated to be constant by controling the grid side converter. The relation between the different currents in the DC-link can be described as

$$
I_{r}-\frac{U_{c}}{R_{1}}-C \frac{\mathrm{d} U_{c}}{\mathrm{~d} t}=I_{o}
$$

where

$$
\begin{gathered}
I_{r}=\frac{3}{4}\left[\begin{array}{ll}
\beta_{r d} & \beta_{r q}
\end{array}\right]\left[\begin{array}{l}
i_{r d} \\
i_{r q}
\end{array}\right], \\
I_{o}=\frac{3}{4}\left[\begin{array}{ll}
\beta_{o d} & \beta_{o q}
\end{array}\right]\left[\begin{array}{l}
i_{o d} \\
i_{o q}
\end{array}\right] .
\end{gathered}
$$

Consequently, the DC link voltage dynamics can be deduced as

$$
\begin{aligned}
\frac{\mathrm{d} U_{c}}{\mathrm{~d} t}= & \frac{3}{4 C} \beta_{r d} i_{r d}+\frac{3}{4 C} \beta_{r q} i_{r q}-\frac{3}{4 C} \beta_{o d} i_{o d} \\
& -\frac{3}{4 C} \beta_{o q} i_{o q}-\frac{U_{c}}{R_{1} C}
\end{aligned}
$$

2.2. Grid side converter modeling. The inverter on the grid side is used to regulate the DC-link voltage. As it is not possible to connect two voltage sources in parallel, which are the inverter and the grid, a filter is required. Furthermore, this later is used to reduce the harmonics around the switching frequency caused by the inverter. The voltages balance across the RL-filter can be expressed as

$$
\begin{aligned}
& V_{o d}=R_{f} i_{o d}+L_{f} \frac{\mathrm{d} i_{o d}}{\mathrm{~d} t}-L_{f} \omega i_{o q}+V_{r d}=\frac{U_{c} \beta_{o d}}{2} \\
& V_{o q}=R_{f} i_{o q}+L_{f} \frac{\mathrm{d} i_{o q}}{\mathrm{~d} t}+L_{f} \omega i_{o d}+V_{r q}=\frac{U_{c} \beta_{o q}}{2}
\end{aligned}
$$

where $V_{o d}$ and $V_{o q}$ are the d-q components of the output inverter voltage and $V_{r d}$ and $V_{r q}$ are the grid voltages in d-q reference frame. $R_{f}$ and $L_{f}$ represent, respectively, the resistance and the inductance on the RL-filter and $\omega$ represents the angular frequency of the RL-filter terminal voltage.

Therefore, the dynamics of the inverter current in $\mathrm{d}-\mathrm{q}$ frame can be deduced from the above equations as

$$
\begin{aligned}
& \frac{\mathrm{d} i_{o d}}{\mathrm{~d} t}=-\frac{R_{f}}{L_{f}} i_{o d}+\omega i_{o q}-\frac{1}{L_{f}} V_{r d}+\frac{U_{c} \beta_{o d}}{2 L_{f}}, \\
& \frac{\mathrm{d} i_{o q}}{\mathrm{~d} t}=-\frac{R_{f}}{L_{f}} i_{o q}-\omega i_{o d}-\frac{1}{L_{f}} V_{r q}+\frac{U_{c} \beta_{o q}}{2 L_{f}} .
\end{aligned}
$$

\section{Control of the grid side converter}

The grid side converter has to maintain the DC-link voltage constant close to its reference signal $\left(U_{c r}=\right.$ $480 \mathrm{~V}$ ) by generating control signals $\beta_{o d}$ and $\beta_{o q}$. The expressions of active and reactive powers on the grid side are respectively given as

$$
\begin{aligned}
& P=V_{o d} i_{o d}+V_{o q} i_{o q}, \\
& Q=V_{o q} i_{o d}-V_{o d} i_{o q} .
\end{aligned}
$$

The inverter is controlled in order to deliver all the active power to the grid while the reactive power is imposed to be adjusted to zero so that the unity power factor is ensured. A phase locked loop (PLL) is needed in order to synchronize the system with the grid phase angle. $V_{o q}$ is set to zero $\left(V_{o q}=0\right)$ so as to adapt equations to synchronous reference frame. Using the q-axis voltage component and its desired reference, a PI controller is applied to generate the desired frequency and the grid phase angle $\theta_{s}$ is then determined by using an integrator. The scheme of the controlled grid side inverter is presented in Fig. 2

Figure 3 shows the block diagram of the PLL. In order to have a null reactive power, the $\mathrm{q}$-axis current component must be regulated as $i_{\text {oqr }}=0$. Besides, we assume that $i_{o d r}=i_{r d}$.

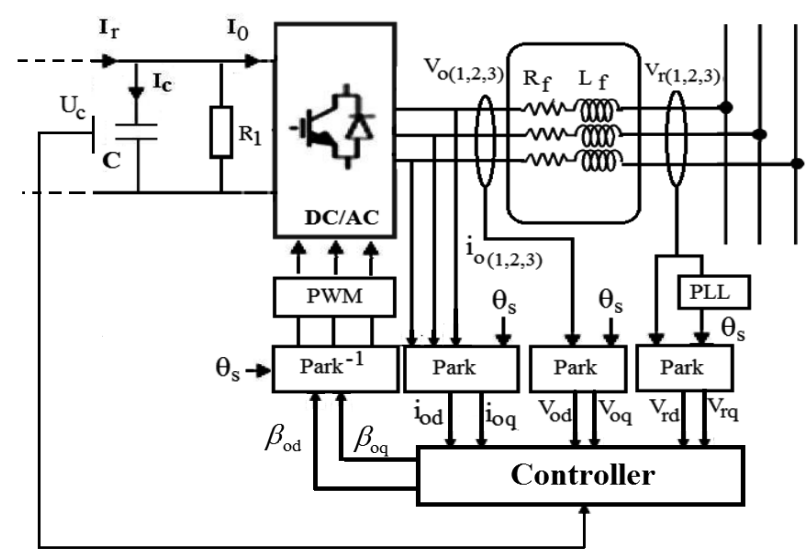

Fig. 2. Diagram of the closed-loop system.

In the next parts, two different fuzzy-based control strategies are proposed in order to control the DC-link voltage. First, a direct Mamdani FLC is applied. Then a fuzzy controller based on T-S fuzzy modeling is proposed.

\section{Mamdani fuzzy logic control}

When the system dynamics are not well known or they comprise nonlinearities, rule base fuzzy logic controllers 


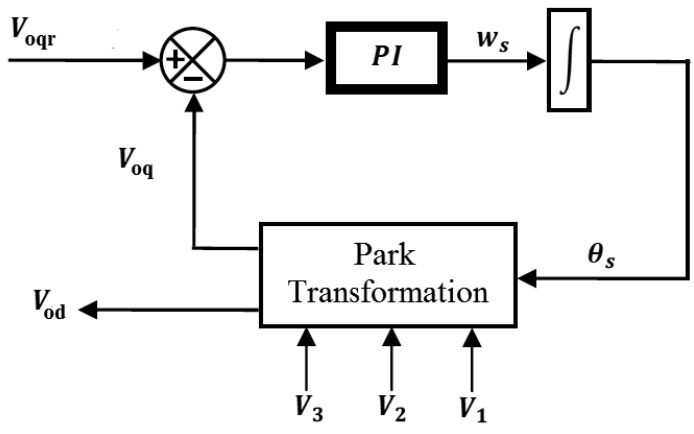

Fig. 3. Diagram of the phase locked loop (PLL).

are useful. Fuzzy logic applies reasoning similar to how human beings choose decisions. Thus, expert knowledge of the system is required to produce fuzzy rules.

The FLC process consists of defining the input variables, setting rules up and computing the output signal from the rules, which is called defuzzification. The proposed blocks find out the control signals based on the error variation in the DC-link voltage and the q-axis component of the current $\left(E_{1}, E_{2}\right)$ and their changes $\left(\Delta E_{1}, \Delta E_{2}\right)$ during a given time horizon. The fuzzy logic process, depicted in Fig. 4 is described in the following.

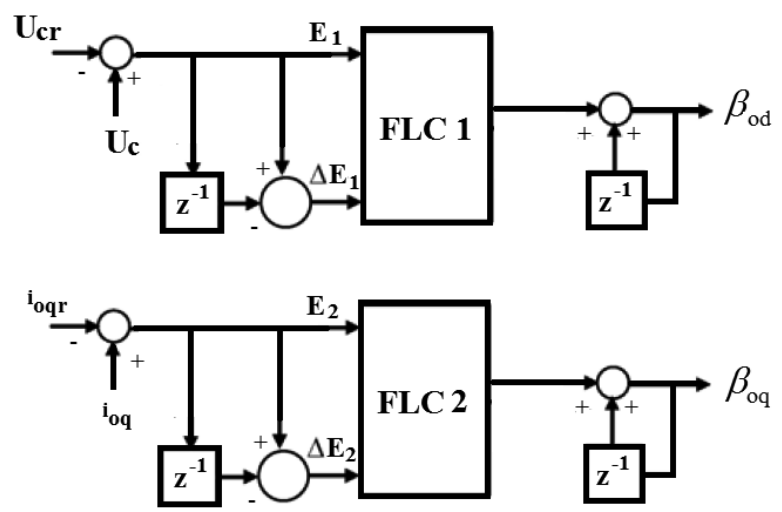

Fig. 4. Diagram of an FLC controlled converter.

4.1. Fuzzification. The designed FLC systems have as input signals

$$
\begin{gathered}
E_{1}=U_{c}-U_{c r}, \\
\Delta E_{1}=E_{1}(k)-E_{1}(k-1), \\
E_{2}=i_{o q}-i_{o q r}, \\
\Delta E_{2}=E_{2}(k)-E_{2}(k-1) .
\end{gathered}
$$

Apart from that, the FLC systems are proposed to generate the control signals $\beta_{o d}$ and $\beta_{o q}$ which are used to produce the switching pulses of the IGBT devices. Triangular membership functions are suitable and adopted for the input and output signals as shown in Figs. 5 and 6 We have considered nine fuzzy sets for both the controllers which allows more sensitivity and accuracy.

The fuzzy sets are denoted as Negative Big (NB), Negative Medium Big (NMB), Negative Medium (NM), Negative Small (NS), zero (Z), Positive Small (PS), Positive Medium (PM), Positive Medium Big (PMB) and Positive Big (PB).

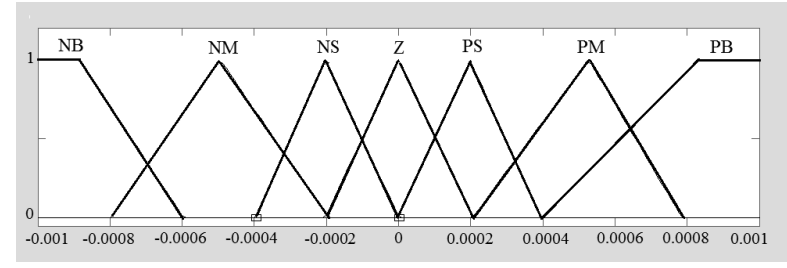

(a)

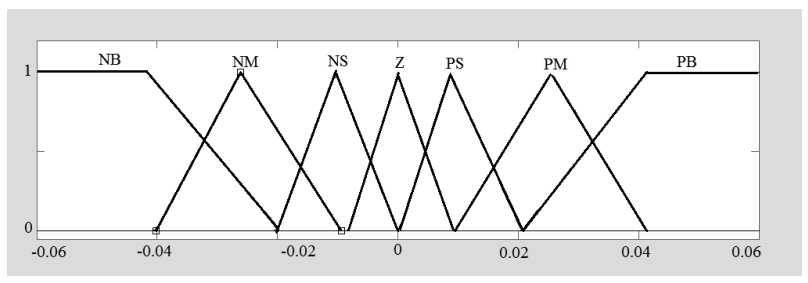

(b)

Fig. 5. Membership functions for FLC system inputs: $E_{1}, E_{2}(\mathrm{a}), \Delta E_{1}, \Delta E_{2}(\mathrm{~b})$

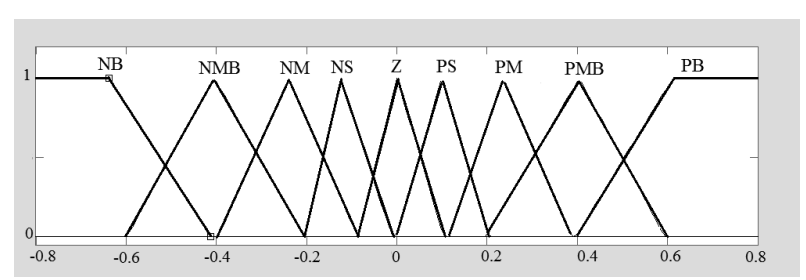

(a)

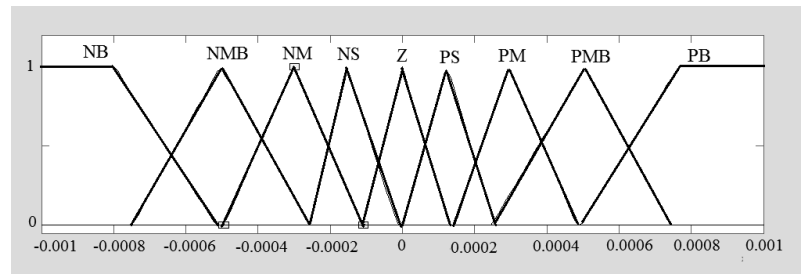

(b)

Fig. 6. Membership functions for FLC systems outputs: $\beta_{o d}$ (a), $\beta_{o q}(b)$.

4.2. Rule base. In total, 49 IF-THEN fuzzy rules are applied to obtain the desired control signals. The fuzzy rules have the following form: 
Table 1. Fuzzy rule base.

\begin{tabular}{|c|c|c|c|c|c|c|c|}
\hline$E_{i} / \Delta E_{i}$ & NB & NM & NS & Z & PS & PM & PB \\
\hline \hline NB & NB & NMB & NM & NM & NS & NS & Z \\
NM & NMB & NM & NM & NS & NS & Z & PS \\
NS & NM & NM & NS & NS & Z & PS & PS \\
Z & NM & NS & NS & Z & PS & PS & PM \\
PS & NS & NS & Z & PS & PS & PS & PM \\
PM & NS & Z & PS & PS & PM & PM & PMB \\
PB & Z & PS & PS & PM & PM & PMB & PB \\
\hline
\end{tabular}

if $E_{1}$ is $\mathrm{NB}$ and $\Delta E_{1}$ is $\mathrm{NB}$, then $\beta_{o d}$ is $\mathrm{NB}$,

if $E_{2}$ is NS and $\Delta E_{2}$ is $\mathrm{NB}$, then $\beta_{o q}$ is NMB.

The fuzzy set domain ranges attributed to the input variables are set as $E_{1}, E_{2}=\left[-10^{-3}, 10^{-3}\right]$, $\Delta E_{1}, \Delta E_{2}=[-0.06,0.06]$, and the output signals are then set as $\beta_{o d}=[-0.8,0.8], \beta_{o q}=\left[-10^{-3}, 10^{-3}\right]$. The entire fuzzy rule base computing the control signals is summarized in Table 1

4.3. Defuzzification. The inference mechanism exploits input fuzzy sets and the corresponding membership values to look up the appropriate rules. Mamdani's min-max method is applied. For defuzzification, we have adopted the center-of-the-gravity method to determine control signals as follows:

$$
\frac{\beta_{o d}}{\beta_{o q}}=\frac{\sum_{i=1}^{n} \mu_{i} c_{i}}{\sum_{i=1}^{n} \mu_{i}}
$$

where $n$ is the number of rules, $\mu_{i}$ represents the membership grade for the $i$-th rule and $c_{i}$ is the coordinate attributed to respective output.

\section{T-S fuzzy control}

A standard fuzzy controller is constructed based on a human expert's knowledge which may not include all situations that occur due to disturbance or noise. Hence, there is a need for a methodology to develop and implement a fuzzy controller in order to ensure its reliability. As a matter of fact, the T-S fuzzy model is capable of accurately approximating any continuous nonlinear system into several local affine models. Indeed, the system closed loop stability may be guaranteed using Lyapunov's theory and disturbance rejection capabilities are ensured since they can be considered in the design unlike the standard fuzzy controller. In the following parts, the proposed T-S fuzzy approach will be detailed.

5.1. T-S fuzzy modeling. Considering the state vector $x(t)=\left[\begin{array}{lll}i_{o d}(t) & i_{o q}(t) & U_{c}(t)\end{array}\right]^{T}$, the control input signal $u(t)=\left[\begin{array}{ll}\beta_{o d} & \beta_{o d}\end{array}\right]$ and the perturbation term $d(t)$, we can describe the studied system by the following state representation:

$$
\dot{x}=A(u) x(t)+B(x) u(t)+B_{d} d(t),
$$

where $A(u)$ is the state matrix, $B(x)$ is the input matrix and $B_{d}$ represents a perturbation matrix of appropriate dimensions, which are respectively given as follows:

$$
\begin{gathered}
A(u)=\left[\begin{array}{ccc}
-\frac{R_{f}}{L_{f}} & \omega & 0 \\
-\omega & -\frac{R_{f}}{L_{f}} & 0 \\
-\frac{3}{4 C} \beta_{o d} & -\frac{3}{4 C} \beta_{o q} & -\frac{1}{R_{1} C}
\end{array}\right] \\
B(x)=\left[\begin{array}{cc}
\frac{U_{c}}{2 L_{f}} & 0 \\
0 & \frac{U_{c}}{2 L_{f}} \\
0 & 0 \\
0 & 0
\end{array}\right] \\
B_{d}=\left[\begin{array}{cc}
1 & 0 \\
-\frac{1}{L_{f}} & 0 \\
0 & -\frac{1}{L_{f}} \\
0 & 0
\end{array}\right] \\
d(t)=\left[\begin{array}{ll}
v_{r d} \\
v_{r q}
\end{array}\right]
\end{gathered}
$$

Since the Takagi-Sugeno fuzzy model allows describing the behavior of a nonlinear system by combining the local linear dynamic subsystems using if-then fuzzy rules, the treated system can be represented by a T-S fuzzy model. Therefore, in order to obtain the corresponding T-S model, three variable premises are chosen as $q_{1}(t)=\beta_{o d}(t), q_{2}(t)=\beta_{o q}(t)$ and $q_{3}(t)=$ $U_{c}(t)$. Hence, the system can be described by eight fuzzy rules, where the $i$-th rule of each fuzzy model takes the following form:

$$
\begin{aligned}
& \text { If } q_{1}(t) \text { is } S_{1 i} \ldots \text { and } q_{3}(t) \text { is } S_{3 i}, \text { then } \\
& \left\{\begin{array}{l}
\dot{x}(t)=A_{i}(u) x(t)+B_{i}(x) u(t)+B_{d} d(t), \\
y(t)=C x(t) .
\end{array}\right.
\end{aligned}
$$


With no loss of generality, we assume that all states are measured. Therefore

$$
C=\left[\begin{array}{lll}
1 & 0 & 0 \\
0 & 1 & 0 \\
0 & 0 & 1
\end{array}\right]
$$

where $S_{i j}$ are fuzzy sets and $A_{i}$ and $B_{i}$ are appropriate subsystem matrices. By replacing each of the variable premises by the corresponding value $q M_{j}=\max \left(q_{j}(t)\right)$ or $q m_{j}=\min \left(q_{j}(t)\right)$ for $j=1, \ldots, 3$ and $i=1, \ldots, 8$, according to the fuzzy rule base, the local subsystem matrices have the following structure:

$$
\begin{gathered}
A_{i}=\left[\begin{array}{ccc}
-\frac{R_{f}}{L_{f}} & \omega & 0 \\
-\omega & -\frac{R_{f}}{L_{f}} & 0 \\
-\frac{3}{4 C} q_{1 i} & -\frac{3}{4 C} q_{2 i} & -\frac{1}{R_{1} C}
\end{array}\right], \\
B_{i}=\left[\begin{array}{cc}
\frac{q_{3 i}}{2 L_{f}} & 0 \\
0 & \frac{q_{3 i}}{2 L_{f}} \\
0 & 0 \\
0 & 0
\end{array}\right] .
\end{gathered}
$$

Then the inferred T-S fuzzy output of the system has the following form:

$$
\left\{\begin{array}{l}
\dot{x}(t)=\sum_{i=1}^{r} \mu_{i}(q(t))\left\{A_{i} x(t)+B_{i} u(t)+B_{d} d(t)\right\} \\
y(t)=C x(t)
\end{array}\right.
$$

For each rule, we define a degree of activation of the membership function by

$$
\mu_{i}(q(t))=\frac{v_{i}(q(t))}{\sum_{i=1}^{r} v_{i}(q(t))}
$$

with $v_{i}(q(t))$ representing the weight to attributed the $i$-th rule, which is given by

$$
v_{i}(q(t))=\prod_{j=1}^{3} S_{j i}(q(t))
$$

The applied membership functions are

$$
\begin{gathered}
f_{a j}=\frac{q_{j}(t)-q m_{j}}{q M_{j}-q m_{j}}, \\
f_{b j}=1-f_{a j} .
\end{gathered}
$$

Table 2 summarizes the parameter setting of each rule.
Table 2. Fuzzy rules base for $i=1, \ldots, 8$.

\begin{tabular}{|c|c|c|c|c|c|}
\hline$S_{1 i}$ & $S_{2 i}$ & $S_{3 i}$ & $q_{1 i}$ & $q_{2 i}$ & $q_{3 i}$ \\
\hline \hline$f_{a 1}$ & $f_{a 2}$ & $f_{a 3}$ & $q M_{1}$ & $q M_{2}$ & $q M_{3}$ \\
$f_{a 1}$ & $f_{a 2}$ & $f_{b 3}$ & $q M_{1}$ & $q M_{2}$ & $q m_{3}$ \\
$f_{a 1}$ & $f_{b 2}$ & $f_{a 3}$ & $q M_{1}$ & $q m_{2}$ & $q M_{3}$ \\
$f_{a 1}$ & $f_{b 2}$ & $f_{b 3}$ & $q M_{1}$ & $q m_{2}$ & $q m_{3}$ \\
$f_{b 1}$ & $f_{a 2}$ & $f_{a 3}$ & $q m_{1}$ & $q M_{2}$ & $q M_{3}$ \\
$f_{b 1}$ & $f_{a 2}$ & $f_{b 3}$ & $q m_{1}$ & $q M_{2}$ & $q m_{3}$ \\
$f_{b 1}$ & $f_{b 2}$ & $f_{a 3}$ & $q m_{1}$ & $q m_{2}$ & $q M_{3}$ \\
$f_{b 1}$ & $f_{b 2}$ & $f_{b 3}$ & $q m_{1}$ & $q m_{2}$ & $q m_{3}$ \\
\hline
\end{tabular}

5.2. Control law. We introduce the state tracking error

$$
e(t)=\left[\begin{array}{c}
i_{o d r}(t)-i_{o d}(t) \\
i_{o q r}(t)-i_{o q}(t) \\
U_{c r}(t)-U_{c}(t)
\end{array}\right]
$$

A new state variable corresponding to an integral action on the error is introduced in order to ensure a smooth tracking of the references parameters $e_{I}=\int e \mathrm{~d} t$.

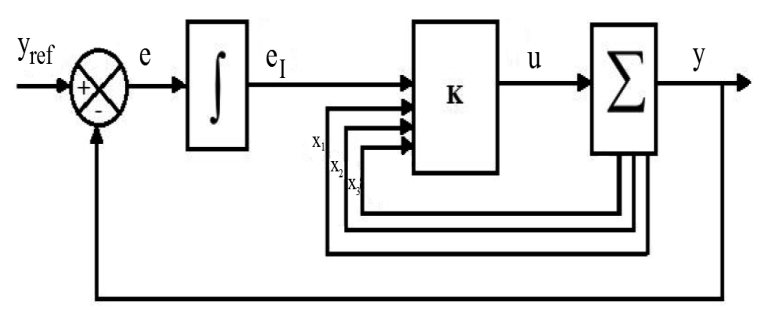

Fig. 7. Diagram of the closed-loop system.

By defining the augmented state vector as $\bar{x}(t)=$ $\left[\begin{array}{ll}x & e_{I}\end{array}\right]^{T}$, the system dynamics can be expressed by the following augmented state model:

$$
\dot{\bar{x}}(t)=\sum_{i=1}^{r} \mu_{i}(q(t))\left(\bar{A}_{i} \bar{x}(t)+\bar{B}_{i} u(t)+\bar{B}_{d} \bar{d}(t)\right),
$$

where the subsystems matrices are

$$
\begin{gathered}
\bar{A}_{i}=\left[\begin{array}{ll}
A_{i} & 0 \\
Q_{1} & 0
\end{array}\right], \\
Q_{1}=\left[\begin{array}{lll}
1 & 0 & 0 \\
0 & 1 & 0 \\
0 & 0 & 1
\end{array}\right], \\
\bar{B}_{i}=\left[\begin{array}{c}
B_{i} \\
0
\end{array}\right], \\
\bar{B}_{d}=\left[\begin{array}{cc}
B_{d} & 0 \\
0 & -I
\end{array}\right] .
\end{gathered}
$$

The augmented perturbation vector is then

$$
\bar{d}(t)=\left[\begin{array}{lllll}
V_{r d} & V_{r q} & i_{o d r} & i_{o q r} & U_{c r}
\end{array}\right]^{T} .
$$


Based on the parallel distributed compensation (PDC) approach, a T-S fuzzy controller is designed. The $i$-th fuzzy controller rule has the following form:

$$
\text { If } q_{1}(t) \text { is } S_{1 i} \ldots \text { and } q_{3}(t) \text { is } S_{3 i} \text {, then }
$$

$$
u(t)=-K_{i} \bar{x}(t) .
$$

Consequently, the global inferred fuzzy controller output is

$$
u(t)=-\sum_{i=1}^{r} \mu_{i}(q(t)) K_{i} \bar{x}(t),
$$

where $K_{i}$ represents the control gain corresponding to each linear submodel.

Hence, substituting (25) in 23), we express the closed-loop model as

$$
\begin{aligned}
\dot{\bar{x}}(t)= & \sum_{i=1}^{r} \sum_{j=1}^{r} \mu_{i}(q(t)) \mu_{j}(q(t)) \\
& \times\left(\left(\bar{A}_{i}-\bar{B}_{i} K_{j}\right) \bar{x}(t)+\bar{B}_{d} \bar{d}(t)\right) .
\end{aligned}
$$

5.3. Stability analysis. The feedback gains of the designed fuzzy controller are obtained by means of an LMI problem. Consider the closed-loop system given in (26.). $H_{\infty}$ performance is applied in order to reject the influence of the perturbation term $\bar{d}(t)$.

The quadratic Lyapunov candidate function $V(\bar{x})=$ $\bar{x}^{T} P \bar{x}$, which is positive definite $\left(P=P^{T}\right)$, has been used in order to verify the system stability and to compute the controller gains. The system is stable if we prove that the Lyapunov function satisfies

$$
\dot{V}(\bar{x}(t))+e_{I}^{T} e_{I}-\gamma^{2} \bar{d}^{T} \bar{d}<0,
$$

where $\gamma$ is a prescribed positive value $(\gamma=0.2)$.

The derivative time of Lyapunov function is

$$
\dot{V}(\bar{x}(t))=\dot{\bar{x}}^{T} P \bar{x}+\bar{x}^{T} P \dot{\bar{x}} .
$$

Substituting the derivative of the augmented state vector, we get the inequality

$$
\begin{array}{r}
{\left[\begin{array}{l}
\bar{x}^{T} \\
\bar{d}^{T}
\end{array}\right]\left[\begin{array}{rr}
\left(\bar{A}_{i}-\bar{B}_{i} K_{j}\right)^{T} P+P\left(\bar{A}_{i}-\bar{B}_{i} K_{j}\right) & P \bar{B}_{d} \\
& 0
\end{array}\right]} \\
\bar{B}_{d}^{T} P \\
{\left[\begin{array}{c}
\bar{x} \\
\bar{d}
\end{array}\right]<0}
\end{array}
$$

Therefore, in order to verify 29 , it suffices to check that

$$
\left[\begin{array}{cc}
\left(\bar{A}_{i}-\bar{B}_{i} K_{j}\right)^{T} P+P\left(\bar{A}_{i}-\bar{B}_{i} K_{j}\right) & P \bar{B}_{d} \\
\bar{B}_{d}^{T} P & 0
\end{array}\right]<0 .
$$

The second term in 27) is developed as

$$
e_{I}^{T} e_{I}-\gamma^{2} \bar{d}^{T} \bar{d}<0
$$

where $e_{I}=\bar{C} \bar{x}$, so we obtain

$$
(\bar{C} \bar{x})^{T}(\bar{C} \bar{x})-\gamma^{2} \bar{d}^{T} \bar{d}<0
$$

with

$$
\bar{C}=\left[\begin{array}{lllllll}
0 & 0 & 0 & 0 & 1 & 0 & 0 \\
0 & 0 & 0 & 0 & 0 & 1 & 0 \\
0 & 0 & 0 & 0 & 0 & 0 & 1
\end{array}\right]
$$

Hence (32) can be written as

$$
\left[\begin{array}{l}
\bar{x}^{T} \\
\bar{d}^{T}
\end{array}\right]\left[\begin{array}{cc}
\bar{C}^{T} \bar{C} & 0 \\
0 & -\gamma I_{1}
\end{array}\right]\left[\begin{array}{c}
\bar{x} \\
\bar{d}
\end{array}\right]<0
$$

which means that we have to check that

$$
\left[\begin{array}{cc}
\bar{C}^{T} \bar{C} & 0 \\
0 & -\gamma^{2} I_{1}
\end{array}\right]<0 .
$$

Combining both conditions resulting from the two terms, we can write

$$
\begin{aligned}
& {\left[\begin{array}{c}
\left(\bar{A}_{i}-\bar{B}_{i} K_{j}\right)^{T} P+\underset{\bar{B}_{d}^{T} P}{P}\left(\bar{A}_{i}-\bar{B}_{i} K_{j}\right)+\bar{C}^{T} \bar{C} \\
\left.P \bar{B}_{d}\right]
\end{array}\right.} \\
& \left.\begin{array}{c}
P \bar{B}_{d} \\
-\gamma^{2} I_{2}
\end{array}\right]<0
\end{aligned}
$$

The problem is finally transformed into an LMI condition that is derived from (35) by using Shur's complement (Carlson et al., 1974) and after multiplying both of sides of (35) by

$$
\left[\begin{array}{cc}
P^{-1} & 0 \\
0 & I
\end{array}\right]
$$

The control gains are therefore deduced as $K_{j}=Y_{j} P$ and satisfy the following LMI:

$$
\left[\begin{array}{cc}
X \bar{A}_{i}^{T}+\bar{A}_{i} X-Y_{j}^{T} \bar{B}_{i}^{T}-\bar{B}_{i} Y_{j} & X \bar{C}^{T} \\
\bar{C} X & -I_{11} \\
\bar{B}_{d}^{T} & 0 \\
& \bar{B}_{d} \\
0 \\
\\
& -\gamma^{2} I_{12}
\end{array}\right]<0
$$

with $X=P^{-1}$ and $Y_{j}=K_{j} X^{-1}$.

\section{Simulation results}

In order to evaluate the performances of the proposed control strategies, a simulation model of the grid side connected inverter has been constructed by using MATLAB/Simulink tools. The parameters of the studied system are given in Table 3 . 
Table 3. System parameters

\begin{tabular}{|c|c|}
\hline Parameter & Value \\
\hline \hline Filter resistance $R_{f}$ & $0.5 \Omega$ \\
Filter inductance $L_{f}$ & $1 \mathrm{mH}$ \\
DC bus capacitance $C$ & $10^{-3} \mathrm{~F}$ \\
DC bus voltage $U_{c}$ & $480 \mathrm{~V}$ \\
Resistance $R_{1}$ & $10^{3} \Omega$ \\
Angular frequency $\omega$ & $100 \pi \mathrm{rad} \cdot \mathrm{s}^{-1}$ \\
Grid frequency & $50 \mathrm{~Hz}$ \\
Grid voltage & $120 \mathrm{~V}$ \\
\hline
\end{tabular}

In order to validate the effectiveness of the designed fuzzy controllers, a resistive load has been inserted and varied at $t=1 \mathrm{~s}$ to check their ability of tracking the reference signal of DC link voltage. The simulation results applying Mamdani FLC are presented in Figs. 8 and 10. Performances related to the T-S fuzzy controller are also shown in Figs. 9 and 11 .

The evolution of the inverter currents $i_{o d}(t)$ and $i_{o q}(t)$ in $\mathrm{d}-\mathrm{q}$ frame using the two controllers is respectively presented in Figs. 8(a) and 9(a) Similarly, the trajectories of voltage $\mathrm{d}-\mathrm{q}$ components are shown in Figs. 8(b) and 9(b) As can be deduced from Figs. 8(c) and 9(c), both Mamdani FLC and T-S fuzzy controllers can provide stable DC-link voltage in spite of the changing load. The tracking error of DC link voltage presented in Figs. 8(d) and 9(d) are somewhat near to zero, which confirms the effectiveness of the designed grid side controllers in tracking the signal reference. However, it can be remarked that the T-S fuzzy controller offers smaller errors compared with the FLC which gives a voltage variation in an acceptable range but less accurately.

It is noticeable that the state signals are tracking the trajectories of their references. The actual inverter d-q axis currents track the reference values and result in a balanced three phase current at the grid as shown in Figs. 10(a) and 11(a) Besides, the three phases voltages obtained at the grid are almost close to $100 \mathrm{~V}$, as presented in Figs. 10(c) and 11(c) For more clarity, we applied a zoom effect as presented in Figs. 10(b), 11(b), 10(d) and 11(d)

Therefore, the fuzzy controlled inverter can successfully achieve the control objective. The performances of both the controllers are analyzed and evaluated. It can be concluded that fuzzy controllers can be a suitable choice for the control of grid connected wind systems. By evaluating the performances of the fuzzy-based control strategies under sudden variation in the resistive load, it can be concluded that the standard FLC has lower current and voltage overshoots at the initial stage. However, the proposed T-S fuzzy controller has the smallest steady voltage error. Therefore, the dynamic DC link voltage obtained using the latter controller shows a slightly better performance although it

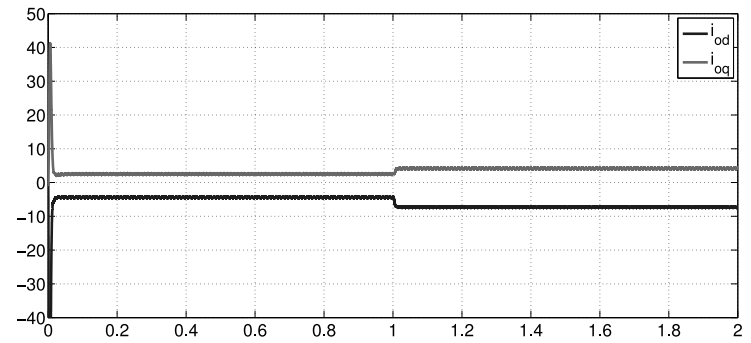

(a)

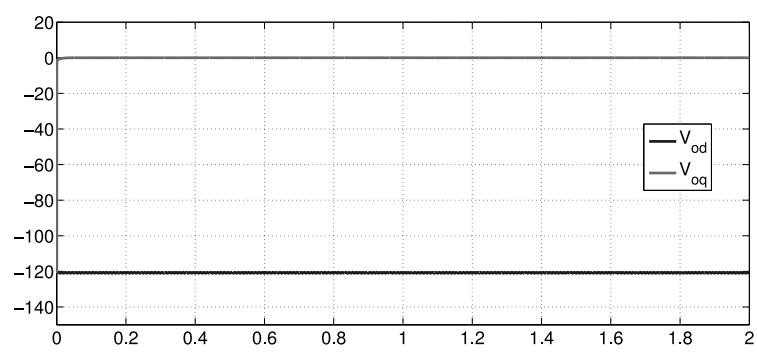

(b)

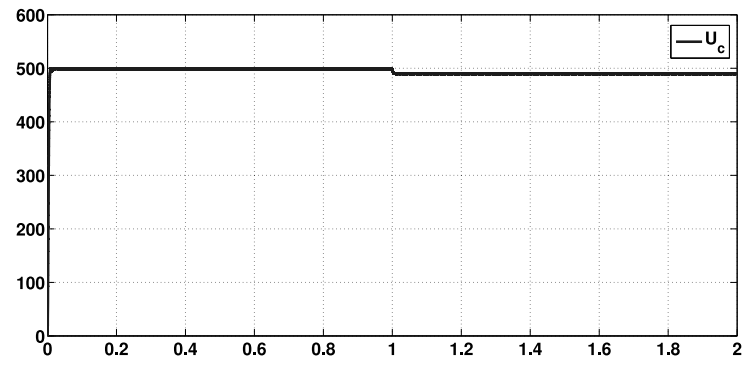

(c)

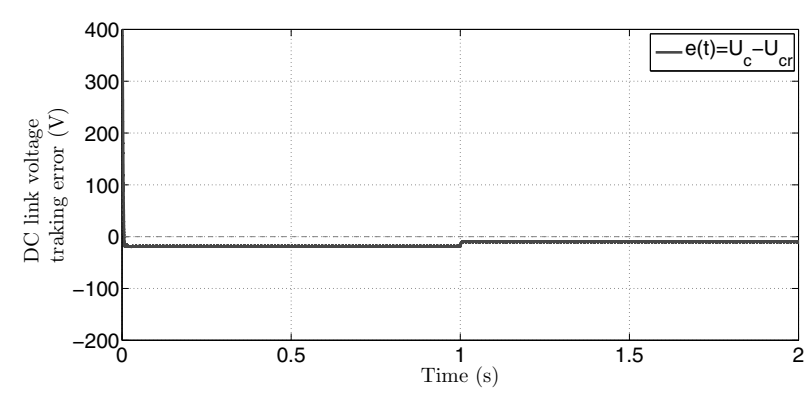

(d)

Fig. 8. Performance of the Mamdani FLC: inverter d-q axis currents (a), inverter d-q axis voltages (b), DC link voltage (c), DC link voltage tracking error (d).

has a more complicated structure. Although it can be seen that the response of both the controllers achieve the main objective, the T-S approach is still more efficient in terms of guaranteeing stability and performance by design. 


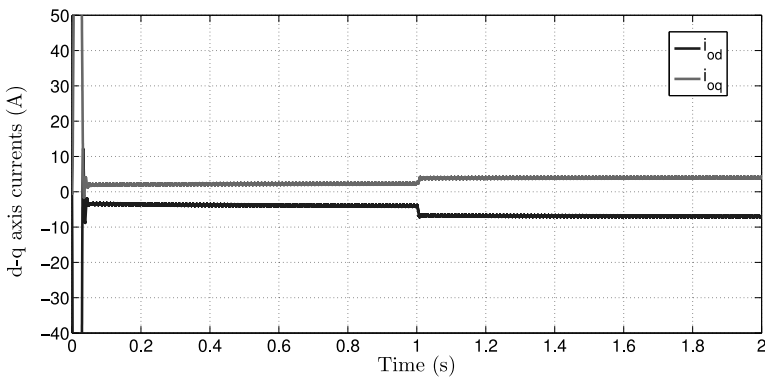

(a)

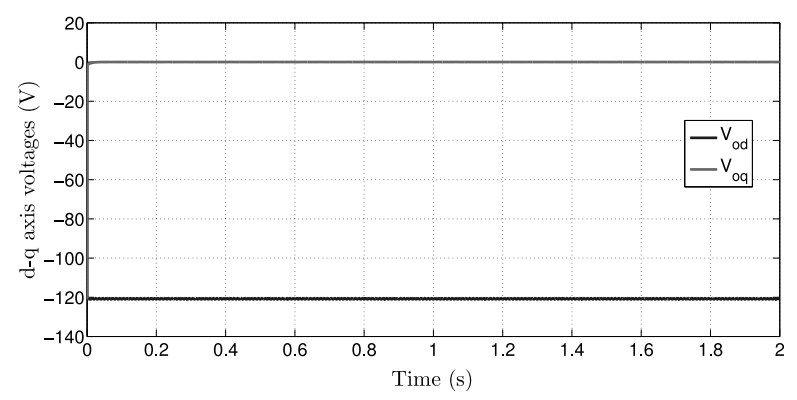

(b)

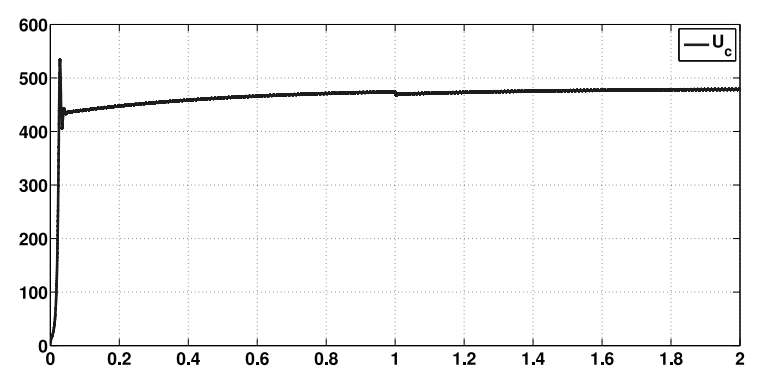

(c)

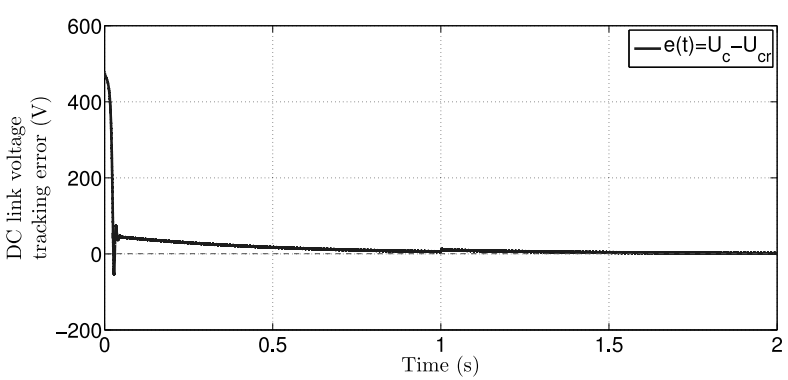

(d)

Fig. 9. Performance of the T-S fuzzy controller: inverter d-q axis currents (a), inverter d-q axis voltages (b), DC link voltage (c), DC link voltage tracking error (d).

\section{Conclusion}

In this study, two control strategies for a grid side converter applied in a wind generation system have been

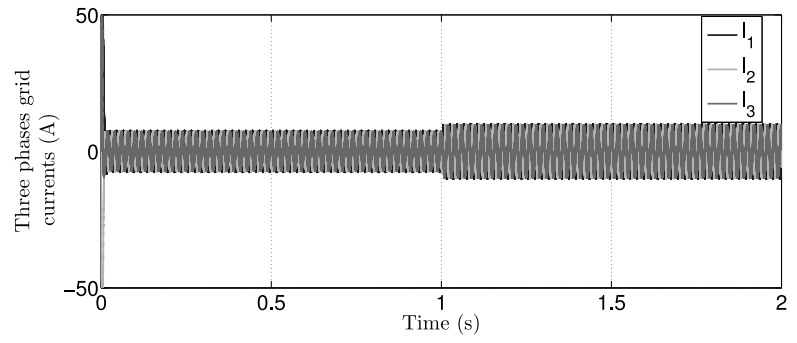

(a)

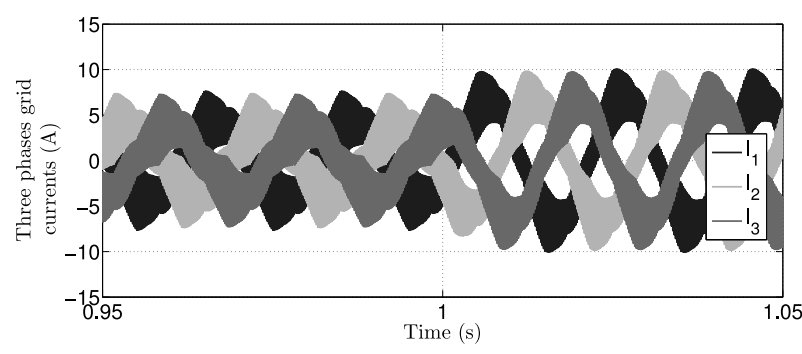

(b)

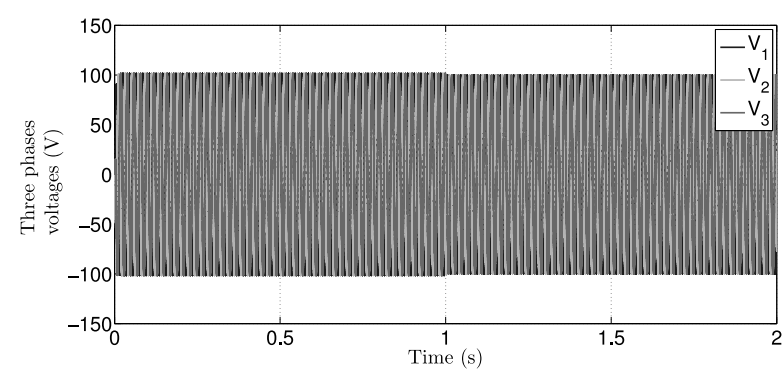

(c)

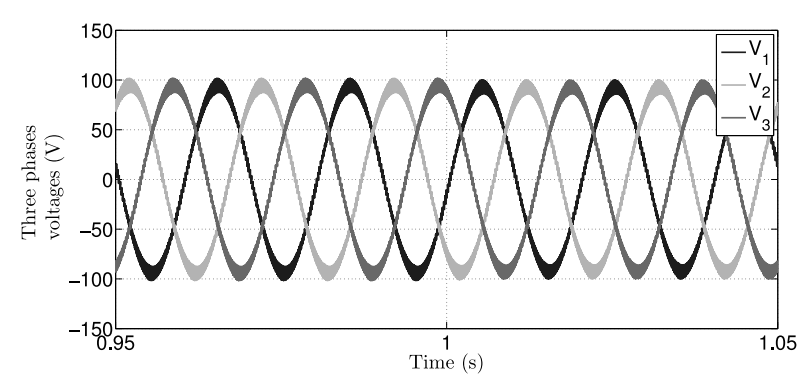

(d)

Fig. 10. Grid currents and voltages using the Mamdani FLCs: grid currents (a), zoom on grid currents (b), grid voltages (c), a zoom on grid voltages (d).

presented and compared. The control objective, which is to regulate the DC link voltage, is satisfactorily achieved using both the controllers and the controlled inverter 


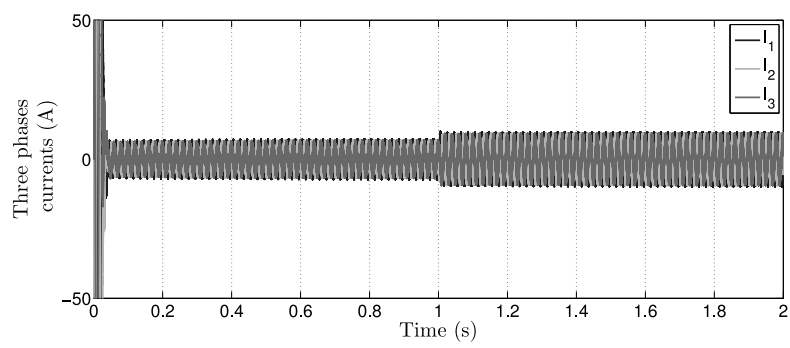

(a)

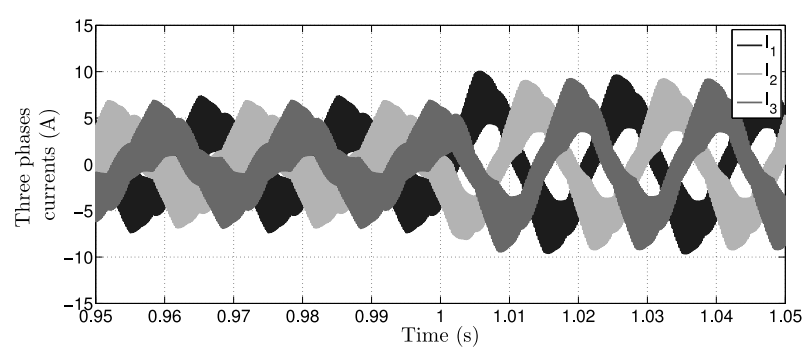

(b)

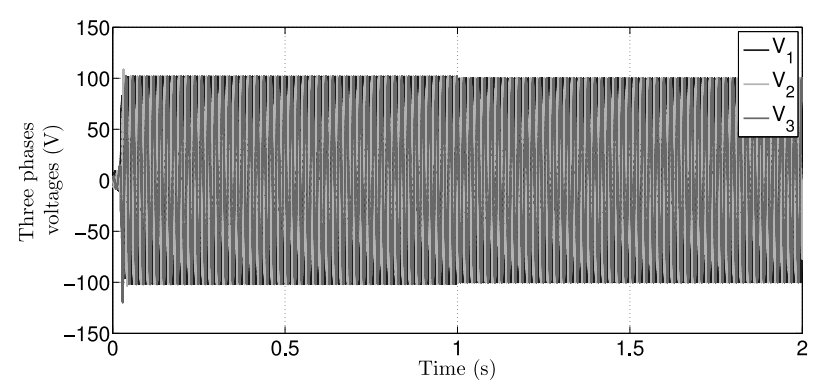

(c)

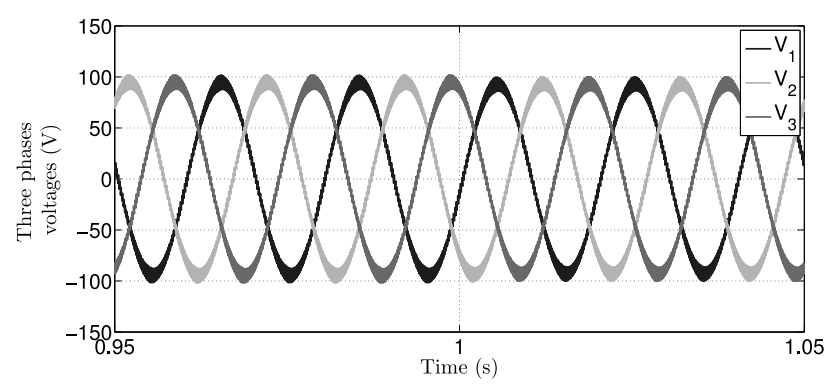

(d)

Fig. 11. Grid currents and voltages using the T-S fuzzy controller: grid currents (a), zoom on grid currents (b), grid voltages (c), zoom on grid voltages (d).

system succeeds in reaching the control objectives. However, the simulation results reveal that the proposed T-S fuzzy approach has better dynamic and steady-state performances since it can ensure stability performances and disturbance rejection. In fact, our results prove that the standard FLC has a lower overshoot in the initial stage. On the other hand, the proposed T-S fuzzy-based control strategy has smaller steady error and may be much more suitable for DC-link voltage control in the WECS. In order to check the performance of the proposed control law, simulation results of both the controllers have been carried out. They show that the proposed controller might bring better performances in wind system applications than classical FLC strategies.

\section{References}

Babu, N.R. and Arulmozhivarman, P. (2013). Wind energy conversion systems-a technical review, Journal of Engineering Science and Technology 8(4): 493-507.

Blaabjerg, F., Liserre, M. and Ma, K. (2012). Power electronics converters for wind turbine systems, IEEE Transactions on Industry Applications 48(2): 708-719.

Boyd, S., El Ghaoui, L., Feron, E. and Balakrishnan, V. (1994). Linear Matrix Inequalities in System and Control Theory, SIAM, Philadelphia, PA.

Camacho, E.F., Samad, T., Garcia-Sanz, M. and Hiskens, I. (2011). Control for renewable energy and smart grids, in $\mathrm{T}$. Samad and A.M. Annaswamy (Eds.), The Impact of Control Technology, Control Systems Society, Copenhagen, pp. 69-88.

Carlson, D., Haynsworth, E. and Markham, T. (1974). A generalization of the Schur complement by means of the Moore-Penrose inverse, SIAM Journal on Applied Mathematics 26(1): 169-175.

Chen, Y.-M., Cheng, C.-S. and Wu, H.-C. (2006). Grid-connected hybrid PV/WIND power generation system with improved DC bus voltage regulation strategy, 21st Annual IEEE Applied Power Electronics Conference and Exposition, APEC'06, Dallas, TX, USA, p. 7.

Chen, Z., Gomez, S.A. and McCormick, M. (2000). A fuzzy logic controlled power electronic system for variable speed wind energy conversion systems, 11th International Conference on Power Electronics and Variable Speed Drives, Sydney, Australia, pp. 114-119.

Chinchilla, M., Arnaltes, S. and Burgos, J.C. (2006). Control of permanent-magnet generators applied to variable-speed wind-energy systems connected to the grid, IEEE Transactions on Energy Conversion 21(1): 130-135.

Chung, I.-Y., Liu, W., Cartes, D.A., Collins, E.G. and Moon, S.-I. (2010). Control methods of inverter-interfaced distributed generators in a microgrid system, IEEE Transactions on Industry Applications 46(3): 1078-1088.

Dixon, J., Contardo, J. and Moran, L. (1997). DC link fuzzy control for an active power filter, sensing the line current only, 28th Annual IEEE Power Electronics Specialists Conference, PESC'97, Saint Louis, MO, USA, Vol. 2, pp. 1109-1114. 
Farh, H.M. and Eltamaly, A.M. (2013). Fuzzy logic control of wind energy conversion system, Journal of Renewable and Sustainable Energy 5(2): 023125.

Goudarzi, N. and Zhu, W. (2013). A review on the development of wind turbine generators across the world, International Journal of Dynamics and Control 1(2): 192-202.

Harrabi, N., Souissi, M., Aitouche, A. and Chaabane, M. (2016). MPPT algorithm for wind energy generation system using TS fuzzy modeling, 5th International Conference on Systems and Control (ICSC), Marrakesh, Morrocco, pp. 157-162.

Harrabi, N., Souissi, M., Aitouche, A. and Chabaane, M. (2015). Intelligent control of wind conversion system based on PMSG using TS fuzzy scheme, International Journal of Renewable Energy Research 5(4): 952-960.

Kadam, D. and Kushare, B. (2012). Overview of different wind generator systems and their comparisons, International Journal of Engineering Science \& Advanced Technology 2(4): 1076-1081.

Kim, Y.-S., Chung, I.-Y. and Moon, S.-I. (2015). Tuning of the PI controller parameters of a PMSG wind turbine to improve control performance under various wind speeds, Energies 8(2): 1406-1425.

Mansour, M., Mansouri, M. and Mmimouni, M. (2011). Study and control of a variable-speed wind-energy system connected to the grid, International Journal of Renewable Energy Research 1(2): 96-104.

Nguang, S.K. and Shi, P. (2006). Robust $h$ infinity output feedback control design for fuzzy dynamic systems with quadratic $d$ stability constraints: An LMI approach, Information Sciences 176(15): 2161-2191.

Nguyen, H.M. and Naidu, D.S. (2011). Advanced control strategies for wind energy systems: An overview, IEEE/PES Power Systems Conference and Exposition (PSCE), Phoenix, AZ, USA, pp. 1-8.

Skretas, S.B. and Papadopoulos, D.P. (2008). Enhanced design and performance of WECS with PMSG connected to MV grid using intelligent control methods, 18th International Conference on Electrical Machines, ICEM 2008, Vilamoura, Portugal, pp. 1-6.

Spooner, E. and Williamson, A. (1996). Direct coupled, permanent magnet generators for wind turbine applications, IEE Proceedings Electric Power Applications 143(1): 1-8.

Tapia, A., Tapia, G., Ostolaza, J.X. and Saenz, J.R. (2003). Modeling and control of a wind turbine driven doubly fed induction generator, IEEE Transactions on Energy Conversion 18(2): 194-204.

Tsoutsos, T.D. and Stamboulis, Y.A. (2005). The sustainable diffusion of renewable energy technologies as an example of an innovation-focused policy, Technovation 25(7): 753-761.

Wang, H.O., Tanaka, K. and Griffin, M.F. (1996). An approach to fuzzy control of nonlinear systems: Stability and design issues, IEEE Transactions on Fuzzy Systems 4(1): 14-23.

Zhang, J. and Cheng, M. (2010). Dc link voltage control strategy of grid-connected wind power generation system, 2nd IEEE International Symposium on Power Electronics for Distributed Generation Systems (PEDG), Hefei, China, pp. 970-975.

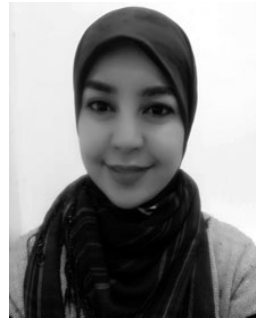

power systems.
Naziha Harrabi obtained a $\mathrm{PhD}$ in automatic control and computer engineering from the $\mathrm{Na}$ tional Engineering School of Sfax (ENIS), University of Sfax, Tunisia, in 2016. She is a member of the Laboratory of Sciences and Techniques of Automatic Control and Computer Engineering (Lab-STA) of the ENIS. Her current research interests include analysis and design of intelligent control systems such as fuzzy control, particularly wind energy, solar energy, and modern

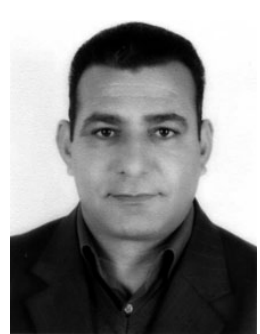

Maher Kharrat obtained the $\mathrm{PhD}$ degree automatic control and computer engineering from the National Engineering School of Sfax (ENIS), University of Sfax, Tunisia, in 2010. He works as an associate professor of automatic control in the Industrial Computing Department of the National School of Electronics and Telecommunications of Sfax (ENET'COM). He is a member of the Laboratory of Sciences and Techniques of Automatic Control and Computer Engineering (LabSTA) of the ENIS. His research interests include systems identification and adaptive control of complex systems, feedback control systems, control theory and subspace identification for linear systems, backstepping and T-S fuzzy control with applications to industrial process.

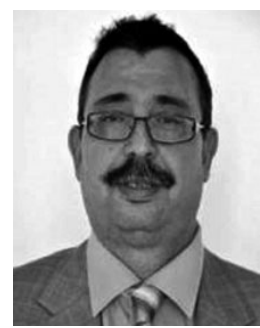

Abdel Aitouche is currently a professor of control engineering and robotics with the Graduate School of Engineering in Lille, France. He is a researcher at CRIStAL (Center of Research on Informatics, Signal and Automation), associated with CNRS (French National Center for Scientific Research). He has authored or coauthored more than 200 papers in journals and conferences. His current research interests include fault-tolerant systems, fault-tolerant control, and model-based fault detection and diagnosis, with their applications in intelligent transportation, process engineering, fermented processes, diesel engine, and renewable energy (PV, wind, fuel cells).

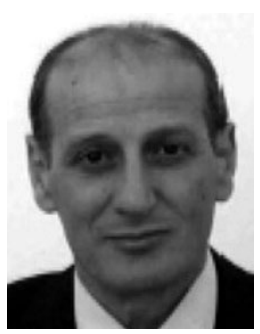

Mansour Souissi received his Doctorat d'État in physical sciences from the University of Tunis, Tunisia, in 2002. He is a professor of automatic control at the Preparatory Institute of Engineers of Sfax, Tunisia. Since 2003, he has been holding a research position at the Automatic Control Unit, National School of Engineers of Sfax, Tunisia. His research interests include robust control, optimal control, fuzzy logic, linear matrix inequalities, and applications of these techniques to agriculture systems and renewable energy systems.

Received: 14 March 2017

Revised: 15 November 2017

Re-revised: 17 January 2018

Accepted: 23 February 2018 